\title{
Double-Layer-Relevant Laboratory Results
}

\author{
D. Diebold, Member, IEEE, C. E. Forest, N. Hershkowitz, Fellow, IEEE, M.-K. Hsieh, \\ T. Intrator, D. Kaufman, G.-H. Kim, S.-G. Lee, and J. Menard
}

\begin{abstract}
Over the past few years, many double-layer-relevant laboratory experiments have been carried out at the Unjversity of Wisconsin. Laboratory stair-step double layers, which resemble three or more weak double layers joined in series, have been produced without ionization for the first time. Doublelayer floating potential fluctuations have been investigated and progress has been made in developing a novel technique for measuring electron energy distribution functions in low-density double layers (i.e., $\lambda_{D} \gg$ probe dimensions). $A$ new inductive plasma source has been developed. With this source, magnetized double layers can be routinely produced. These magnetized double layers are often weak stair-step double layers that are oblique to the magnetic field. Laboratory data of emitting probe characteristics taken in tenuous plasmas have helped to quantify space-charge-enhanced plasma gradient induced error in doublelayer electric field measurements made by satellite double probes. Also, magnetic sheaths have been experimentally studied and compared with theory.
\end{abstract}

\section{INTRODUCTION}

A LTHOUGH it has long been thought that double layers (DL's) may be responsible for the acceleration of charged particles associated with the aurora borealis, it is still not clear whether it is strong double layers (SDL's), i.e., DL's with $e \Delta \phi \gg 10 T_{e}$, where $\Delta \phi$ is the potential drop across the double layer and $T_{e}$ is the plasma electron temperature, or weak double layers (WDL's), i.e., DL's with $e \Delta \phi \leq 10 T_{e}$, that are primarily responsible for auroral particle acceleration. Temerin et al. [1] found that probes on board the S3-3 polarorbiting satellite observed many WDL's, between altitudes of 6000 and $8000 \mathrm{~km}$, aligned with and moving along the magnetic field. They proposed that a series of these DL's might account for a large portion of the parallel potential drop that accelerates auroral particles along field lines. More recently, but again from S3-3 data, Mozer [2], [3] has found evidence of field-aligned SDL's and has suggested that SDL's may be primarily responsible for auroral particle acceleration.

Analysis of Viking data by Boström et al. [4]-[6] revealed a predominance of WDL's in regions of relatively weak overall potential drop, i.e., less than $1 \mathrm{keV}$ as inferred from particle data. Mozer [3] has noted in his analysis of S3-3 data that when S3-3 was in regions of strong overall potential drop (roughly as much as $10 \mathrm{keV}$ or more as inferred from particle

Manuscript received February 18, 1992; revised May 29, 1992.

D. Diebold, C.E. Forest, N. Hershkowitz, T. Intrator, G.-H. Kim, S.-G Lee, and J. Menard are with the Department of Nuclear Engineering and Engineering Physics, University of Wisconsin-Madison, Madison, WI 53706. M.-K. Hsieh is with the Department of Electrical and Computer Engineering, University of Wisconsin-Madison, Madison, WI 53706.

D. Kaufman is with the Department of Mechanical Engineering, University of Wisconsin-Madison, Madison, WI 53706.

IEEE Log Number 9205361. data) its data were dominated by SDL's and that when S3-3 was in regions of weak overall potential drop its data were dominated by WDL's. Although Viking has flown in both these types of regions, the analysis of Viking data to date has been concentrated on those regions of weak overall potential drop [6]. It may be that in regions of weak overall potential drop WDL's are primarily responsible for charged particle acceleration while in regions of strong potential drop it is SDL's that are primarily responsible [3], [6].

Our group has been experimentally addressing DL issues for the last decade at the University of Wisconsin, and before that at the University of Iowa. This laboratory work has included both SDL's [7]-[11] and WDL's [12]-[22]. More specifically, this work includes the first laboratory observations of ion-acoustic-type DL's [18], slow ion acoustic DL's [20], two-step DL's [16], very weak DL's (i.e. $\Delta \phi \simeq T_{e}$ ) [18], DL's with no external electric field applied [13], currentless DL's [20], stair-step DL's [21], magnetized stair-step DL's [22], SDL's in a triple plasma device [7], WDL's in a triple plasma device [12], and U-shaped DL's in a magnetic field in a triple plasma device [8]. It should be noted that all our DL's have been produced in triple plasma devices [9] and that with the exception of the DL's described in [10], [18], and [20], these DL's have been stationary. For a review of many of these, as well as other DL experiments, the reader is referred to Hershkowitz [23].

\section{STAIR-STEP DOUBLE LAYERS}

Chan and Hershkowitz [16] were the first to show that it is possible to obtain laboratory two-step DL's (which resemble two single WDL's joined in series) without ionization occurring in the DL's. An example of a two-step DL, measured by Bailey, is shown in Fig. 1. Chan and Hershkowitz [16] argued that two-step DL's can be produced when the ratio $\lambda_{D} / L$, where $\lambda_{D}$ is the Debye length and $L$ is the length of the chamber in which the DL's are produced, is made to be less than approximately $1 \times 10^{-2}$. In their experiment, they achieved this both by increasing the plasma density and by increasing $L$. However, subsequent efforts in which both the plasma density and the device length were further increased failed to produce stair-step DL's with three or more single DL's joined in series.

Bailey and Hershkowitz [21] were the first to achieve, without ionization, laboratory stair-step DL's with three or more single WDL's joined in series. These stair-step DL's were achieved by careful control of the boundary conditions and appeared to be a new class of laboratory DL's that were intermediate between anomalous resistivity [24] and 


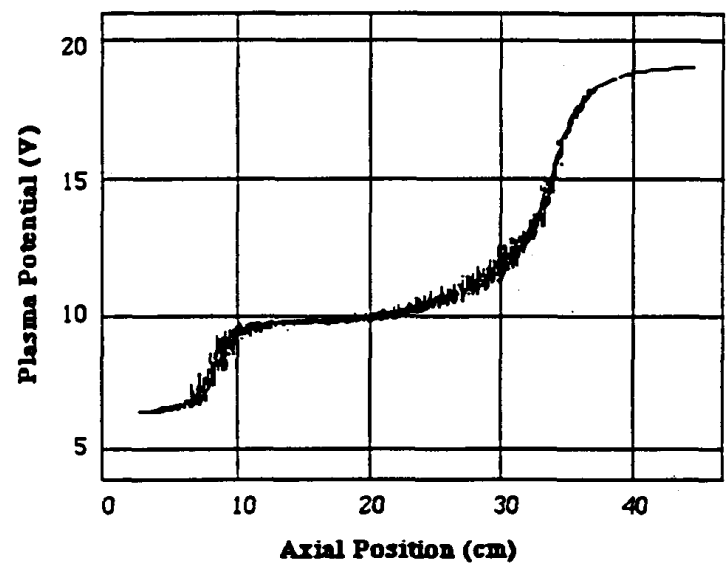

Fig. 1. Plasma potential (volts) versus axial position (cm) of a two-step DL.

conventional BGK [25], [26] DL's. The stair-case DL's also appeared to depend on turbulence for their existence. As an extension of this work, Forest and Kaufman studied the highfrequency floating potential fluctuations, as measured by a capacitive probe [27], associated with these and other DL's. An example of their data is shown in Fig. 2. Some qualitative correspondence between the high-frequency floating potential fluctuations and DL's was found. For example, in Fig. 2(a) there is a peak in fluctuation amplitude at a frequency of approximately $45 \mathrm{MHz}$ on the high-potential side of the DL shown in Fig. 2(b) and another peak at a frequency of approximately $25 \mathrm{MHz}$ on the low-potential side of that DL.

In an effort to determine whether high-frequency fluctuations associated with DL's caused thermalization of electron beams created by DL's, Kaufman, Forest, and Lee attempted (unsuccessfully) to measure the electron distribution functions of stair-step DL's. Standard, for our laboratory, 7-mmdiameter Langmuir probes were found to function poorly in the DL's which were investigated. In particular, probe $I-V$ (current versus voltage) characteristics did not show knees, which are a basic feature of probe $I-V$ characteristics.

It was first thought that the absence of knees was due to probe surface contamination. A pulsed probe technique developed by Szuszczewicz and Holmes [28] (also see Holmes and Szuszczewicz [29]) was employed. This technique can considerably reduce surface-contamination-induced distortion of probe $I-V$ characteristics. However, it should be noted that with this technique a nonzero, constant contact potential difference between the voltage applied to a probe and the voltage on the surface (which interacts with the plasma) of that probe can exist. Therefore, plasma potential cannot be inferred from data obtained by this technique. This was not a concern for Kaufman and Lee, however, as they were able to measure plasma potential with emissive probes. Some typical data taken by Kaufman and Lee with the pulsed probe technique are shown in Fig. 3. As is evident from the data, a knee still could not be found.

In a second attempt to obtain probe characteristics with knees, guarded Langmuir probes were employed. In the double layers which Kaufman and Lee investigated, $n \sim 10^{6} \mathrm{~cm}^{-3}$

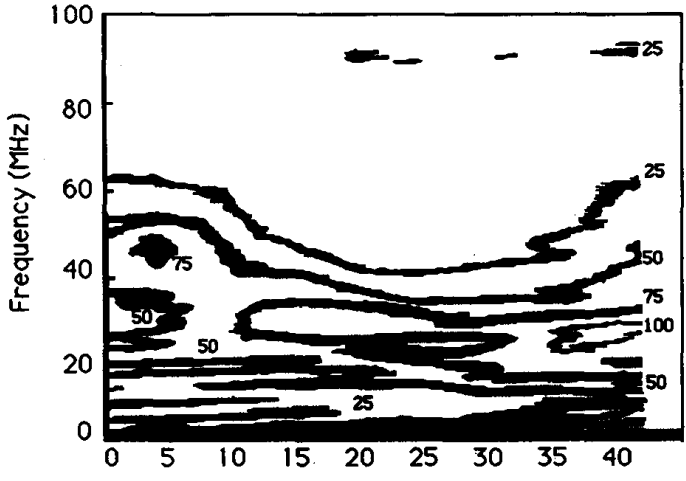

(a)

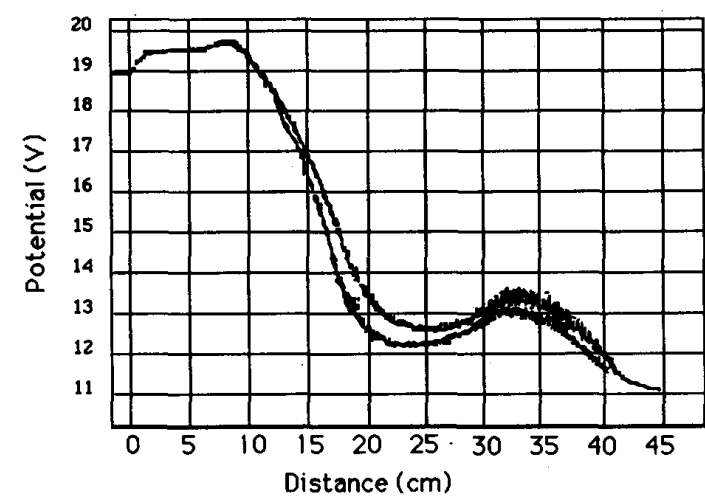

(b)

Fig. 2. Capacitive probe measurements of the high-frequency floating potential fluctuations associated with a DL. (a) Equiamplitude contours (marked in $\mathrm{dB}$ ) in the frequency $(\mathrm{MHz})$, distance $(\mathrm{cm})$ domain. (b) Plasma potential (volts) versus distance $(\mathrm{cm})$ of the corresponding DL.

and $T_{e} \simeq 2 \mathrm{eV}$, which means that $\lambda_{D}$ was approximately $1 / 3$ of the probe diameter. In low-density plasmas where $\lambda_{D}$ is comparable to the probe diameter, the sheaths of even planar probes become spherical, and the $I-V$ characteristics of probes with spherical sheaths have no knees [30]. When plasma density becomes low, the fringing fields of a guarded probe's sheath should fall mainly on its guard; i.e., for decreasing density, the effective area of the probe should remain roughly constant while the effective collecting area of its guard increases. Insulating material is used to electrically isolate the probe and its guard. The guard and probe are kept at the same voltage by separate power supplies so that the guard's current does not add to the probe's current.

The qualitative design of the first guarded probe employed by Kaufman and Lee is shown in Fig. 4(a). Characteristics from this probe still showed no knee. The guarded probe shown qualitatively in Fig. 4(b) was then tried and yielded $I-V$ characteristics with knees. It is believed that the important difference between the guarded probes shown in parts (a) and (b) of Figs. 4 is that insulating surfaces are exposed to the plasma for the probe shown in Fig. 4(a) but not the probe shown in Fig. 4(b). Insulating surfaces are known to charge negative with respect to the plasma potential; they charge toward the floating potential. In low-density plasmas, sheaths 


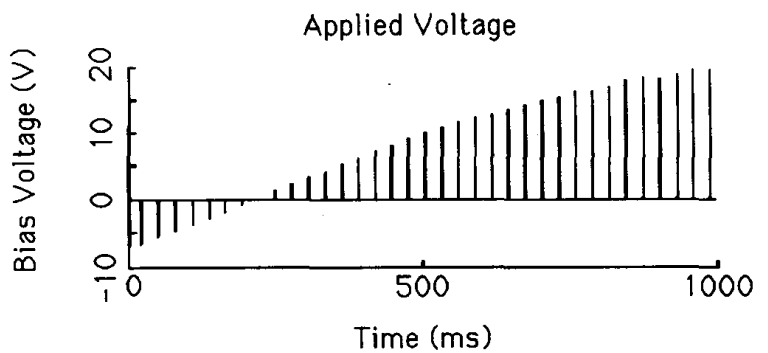

(a)

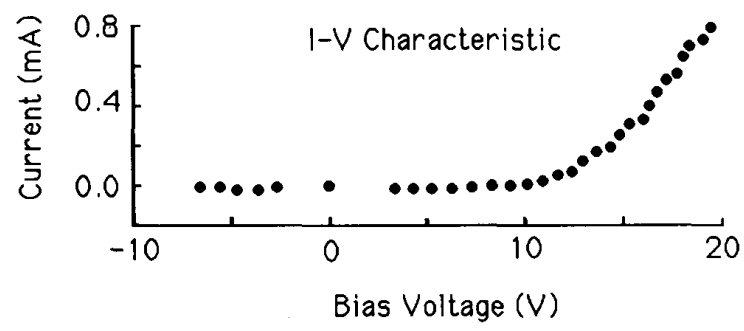

(b)

Fig. 3. Data from a probe in a plasma when the voltage applied to the probe was pulsed. (a) Pulsed bias voltage (volts) applied to a probe versus time (ms). (b) The corresponding probe current (milliamps) versus bias voltage (volts), also referred to as the probe's $I-V$ characteristic, using the pulsed probe technique.
TOP VIEW

ai.

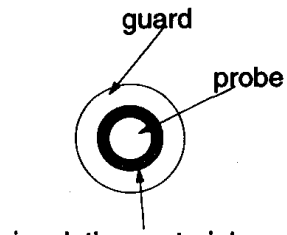

insulating material

bi.

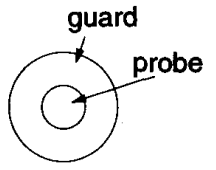

SIDE VIEW

aii.

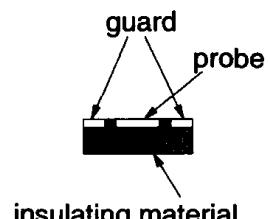

bii.

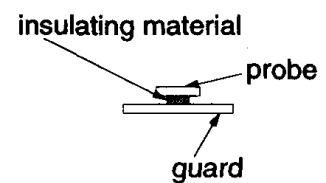

Fig. 4. Schematics of the guarded probes used by Kaufman and Lee. The thicknesses in the side views are exaggerated for clarity. The first design tried is shown in (a) and the second in (b).

can become quite large; and it is believed that the probe shown in Fig. 4(a), and perhaps its guard as well, were in the sheath of the insulating material.

Fig. 5 depicts a "witch's hat". One might think of the witch's hat as a guarded probe with a large guard that is bent into a "hat" over the probe. Again, the hat and probe are biased with different power supplies so that the hat's current does not add to the probe's current. However, with the witch's hat, the hat is biased to the local plasma potential (as determined by an emissive probe) rather than the probe's voltage. If the hat is successful in containing the probe's sheath within it, then there is no sheath between the hat and the plasma. Under such

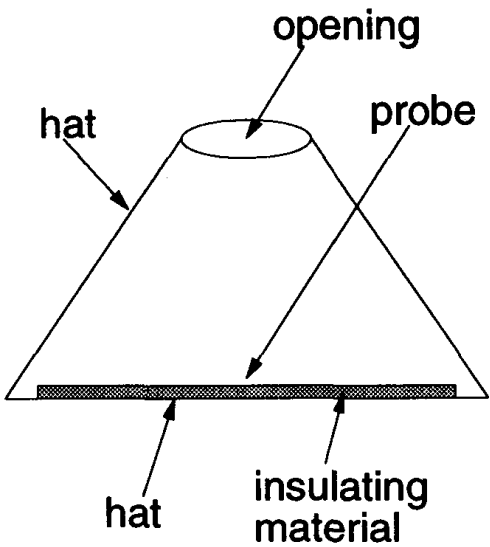

Fig. 5. Schematic of the witch's hat.

conditions, the effective collecting area of the probe is just the area of the opening in the top of the hat.

Probe characteristics with knees were obtained with the witch's hat and the knees were at the same probe bias voltage as the knees obtained with the guarded probe. However, the electron energy distribution functions measured by the two probes were not the same; the effective electron temperatures measured by the witch's hat were greater than those measured with the guarded probe. Furthermore, the voltage bias at which the knees occurred did not change as the probe was moved axially through the double layer chamber. Subsequent emissive probe measurements showed that double layers were destroyed when either the witch's hat or the guarded probe was used. At this point, the effort to measure the electron energy distribution functions associated with double layers was abandoned.

\section{DOUBle LAYERS OBlique to AN APPLIED MAGNETIC FIELD}

In the laboratory, constraints are imposed on the production of DL's by the plasma sources that are used. Consequently, there has been much work in our laboratory regarding plasma source development [7], [31]-[41]. In particular, in the past, plasma source limitations have restricted our efforts to investigate DL's which are oblique to externally applied magnetic fields. There has been interest in such DL's, among other reasons, because when Temerin et al. [1] suggested that auroral particle acceleration might be due a series of WDL's aligned with the magnetic field, they also suggested that these WDL's might be two-dimensional in structure.

Most of our double layers are obtained in the absence of applied magnetic field. Our plasmas are usually produced with hot, electron-emitting, thoriated tungsten filaments. When a magnetic field is applied, the emitted electrons tend to be confined to those field lines which intersect the discrete filaments. Hence, in the presence of an applied magnetic field, our filament discharges tend to be noisy and have large spatial variations perpendicular to the applied magnetic field [8], [23]. Despite these problems, Coakley et al. [8] did manage to produce DL's in a magnetic field of about 40 gauss. 
For the reasons discussed above, it is difficult when using filament sources to obtain DL's in magnetic fields. Q machines have been used to generate DL's in magnetic fields. However, with regard to studying WDL's that are oblique to the magnetic field, $\mathrm{Q}$ machines have the disadvantage that $T_{e}=T_{i} \simeq$ $0.2 \mathrm{eV}$, which means that it is not possible to produce ionacoustic-type WDL's with Q machine plasmas.

Intrator et al. [22] have developed an inductive source that produces relatively quiet and spatially uniform plasmas with $T_{e}(\sim 3 \mathrm{eV}) \gg T_{i}(\sim 0.1 \mathrm{eV})$ in magnetic fields of up to $360 \mathrm{~g}$. To produce WDL's oblique to applied magnetic fields, Intrator et al. [22] used two such inductive sources in the two source chambers of the triple plasma device double layer investigator-II (DOLI-II), which has been described elsewhere by Intrator et al. [42].

With argon as the working gas, plasma was produced with two loop antennas. One antenna was powered with RF at $13.56 \mathrm{MHz}$ and the other antenna was powered with RF at approximately $10 \mathrm{MHz}$. In the target chamber, where the DL's were produced, the plasma density was approximately $5 \times 10^{9} \mathrm{~cm}^{-3}$ when an external field of $360 \mathrm{~g}$ was applied.

One interesting feature of this type of inductive source is that the plasma potentials in both the sources and the target oscillate at the RF input power frequencies. The RF frequencies employed are great enough that ions and the emissive probes used to measure the plasma potential cannot respond to plasma potential fluctuations at the RF frequency. On the other hand, the electrons have enough mobility that they can respond to plasma potential fluctuations at the RF frequency. Hence, during part of the RF cycle of each source, electron beams traveling parallel to the magnetic field can exist while during the other part of the RF cycle antiparallel electron beams can exist. This is similar to the situation implied by satellite data analyzed by Hultqvist et al. [43], which suggested the simultaneous (to within the time resolution of their diagnostics) existence of upward moving, magnetic-fieldaligned electron and ion beams.

Stair-step DL's were produced by Intrator et al. [22] with up to four (or possibly more) WDL's in series, oblique to the magnetic field. The average perpendicular scale length of DL's produced by Intrator et al. [22] was found to be dependent on the magnetic field and roughly proportional to the inverse of the magnetic field strength (or ion gyroradius). The corresponding average parallel scale length was found to be only marginally dependent on magnetic field.

Fig. 6 is an example of data taken by Intrator et al. [22] and shows two-dimensional equipotential contours taken in the target chamber when a $180 \mathrm{~g}$ field was applied. In Fig. 6 , DL's are those regions in which the equipotential contours are closely spaced. Note that if the potential shown in Fig. 6 had only been measured along a line (similar to satellite data, which are only measured along the satellite's path) instead of in two dimensions, then the number of DL's that would have been measured would have depended on the line of measurement. For example, if the data had been measured along the line segment between the points $(r, z)=(6,-20)$ and $(-6,30)$, only one DL (the DL in the region near $(5,-10)$ ) would have been measured. On the other hand, if the data

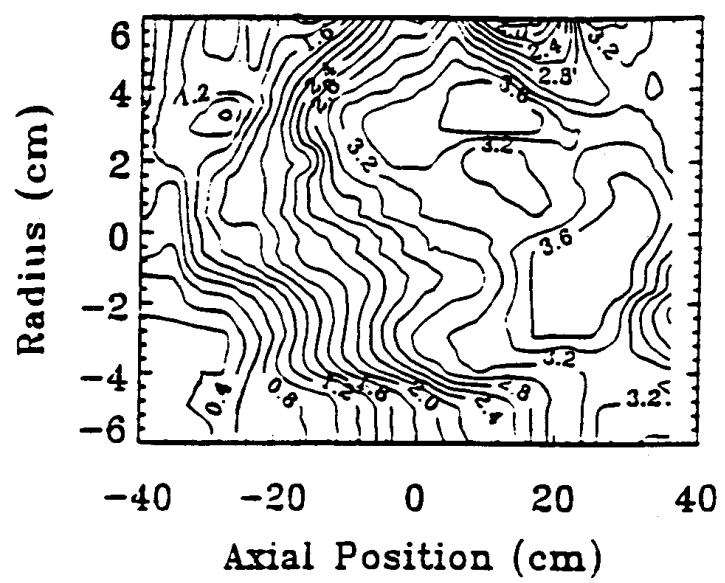

Fig. 6. Equipotential contours (in volts) in the radius $(\mathrm{cm})$, axial position $(\mathrm{cm})$ domain. The applied magnetic field was $180 \mathrm{~g}$ and in the axial direction.

had been measured along the line segment between $(0,-40)$ and $(6,20)$, then three DL's (one near the point $(0.5,-30)$, another near $(2,-12)$, and the last near $(5,14))$ would have been measured. For more details regarding this experiment, the reader is referred to Intrator et al. [22].

\section{SPACE-Charge-Enhanced Plasma GRADIENT ERROR}

While plasma sources place limits on the laboratory DL's that we can produce, plasma diagnostics place limits on the accuracy with which we can measure DL's. We use probes (collecting, emitting, capacitive, etc.) extensively in our laboratory; therefore, in addition to plasma source development, we have done much work on the development of these probes and the techniques with which to use them [44]-[57]. For a review of these and other probe-related works, the reader is referred to Hershkowitz and Cho [58], Hershkowitz et al. [59], and Hershkowitz [60].

As part of our laboratory's probe-related effort, Diebold et al. [56] measured (thermionically) emitting probe characteristics in tenuous laboratory plasmas with densities very roughly in the range of $10^{3} \mathrm{~cm}^{-3}$ to $10^{5} \mathrm{~cm}^{-3}\left(T_{e} \simeq\right.$ $1-2 \mathrm{eV})$. The data from these laboratory measurements helped Diebold et al. [56] in their efforts to quantify the "spacecharge-enhanced, plasma gradient induced error (PGIE)" as it relates to photoemitting, current biased satellite double probe measurements of magnetospheric double-layer electric fields.

As example of such measurements, consider double probe measurements made by Viking of WDL's. Boström et al. [4] have reported Viking data in which small-scale $(\sim 100 \mathrm{~m}$, compared with the $80 \mathrm{~m}$ distance between the two probes of the Viking double probe), large-amplitude $(|\Delta n| / n \leq$ $50 \%$, negative-potential $(|\Delta \phi| \leq 2 \mathrm{~V})$ solitary waves moved upwards along the magnetic field. These structures, which resembled ion holes, often had a net potential drop $(\leq 1 \mathrm{~V})$ in the upward direction and were interpreted to be WDL's. It is under conditions such as these, when there can be a relatively large difference in the plasma conditions at the two 


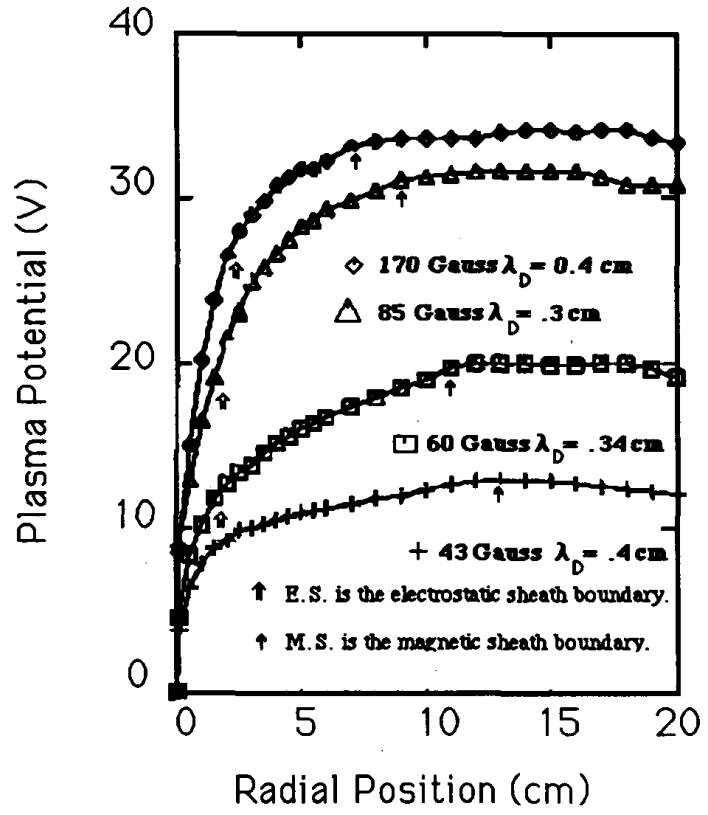

Fig. 7. Plasma potential (volts) versus radial position $(\mathrm{cm})$ of the sheaths near a planar electrode in a He plasma for various applied magnetic fields and plasma conditions. The angle $\psi$, between the applied magnetic fields and the normal to the planar electrode was $60^{\circ}$. The solid arrows indicate the beginning of the magnetic presheath and the open arrows indicate the beginning of the electrostatic sheath.

probes of a double probe, that one might expect space-chargeenhanced PGIE. Furthermore, if space-charge-enhanced PGIE were significant, one would expect it to cause an error that is qualitatively similar to the measurements reported by Boström et al. [4].

Diebold et al. [56] were able to quantify space-chargeenhanced PGIE. They found that, for the data reported by Boström et al. [4], space-charge-ẹnhanced PGIE was not significant. For more details concerning this work, as well as an explanation of what space-charge-enhanced PGIE is and the quantitative expressions describing it, the reader is referred to [56].

\section{MAgNeTIC SHEATHS}

In our laboratory, we have studied many nonneutral plasma phenomena which are not commonly referred to as DL's. Our investigations of plasma wakes [61], [62], plasma expansions [63], [64], and the virtual cathodes near electron-emitting cathodes in plasmas [65] have found potential steps associated with two adjacent regions of net electrical charge, one region of net electrons and one region of net ions. In this sense, these investigations also have been studies of DL's. On the other hand, the sheaths of nonemitting objects [66]-[68] are regions of net ions only; there are no regions of net electrons adjacent to sheaths.

Kim et al. [69] measured the sheath potential structure of an nonemitting object in the presence of an oblique magnetic field. In their experiment, up to $500 \mathrm{~W}$ (at $13.56 \mathrm{MHz}$ ) was inductively coupled into He plasmas in the presence of a maxi- mum applied magnetic field of $170 \mathrm{~g}$. Plasma densities were in the $10^{7} \mathrm{~cm}^{-3}$ range, electron temperature was approximately 5 to $8 \mathrm{eV}$, and ion temperature was $\leq 0.5 \mathrm{eV}$.

The sheath of the electrode was found by Kim et al. [69] to consist of the usual electrostatic sheath (which scaled with the electron Debye length) and a magnetic presheath. Fig. 7 shows data taken by Kim et al. [69]. The arrows indicate the beginning of the magnetic presheath (solid arrows) and the beginning of the electrostatic presheath (open arrows).

In rough agreement with a theory by Chodura [70], the magnetic presheath length was found to be approximately proportional to the ion gyroradius (or the inverse of the magnetic field), the ion acoustic sound speed (i.e., $C_{s}=$ $\left(T_{e} / M_{i}\right)^{1 / 2}$ rather than $\left.v_{i, t h}=\left(T_{i} / M_{i}\right)^{1 / 2}\right)$, and $\sin \psi$, where $\psi$ is the angle between the magnetic field direction and the normal to the planar electrode. In addition to fusion devices with large magnetic fields nearly parallel to their walls, this work may be relevant to satellites and satellite probes in magnetic fields.

\section{ACKNOWLEDGMENT}

The authors would like to thank C. Chan, A. D. Bailey III, M-H. Cho, and J. DeKock for their contributions to the doublelayer investigations. Thanks are also due to J. Meyer for his assistance in preparing the figures.

\section{REFERENCES}

[1] M. Temerin, K. Cerney, W. Lotko, and F.S. Mozer, "Observation of double layers and solitary waves in the auroral plasma," Phys. Rev. Lett., vol. 48, p. $1175,1982$.

[2] F.S. Mozer, "Questions about the electric fields in the auroral acceleration region," in Abstracts Workshop Plasma Experiments in the Laboratory and in Space (Alpbach, Austria), July 1-5, 1991, p. 25,

[3] F. S. Mozer, "Parallel electric fields," in Abstracts AGU Chapman Conf. Auroral Plasma Dynamics (convened by R. L. Lysak, Minneapolis), Oct. $21-25,1991$, p. 25 ; and the discussion immediately following this talk, 1991.

[4] R. Boström et al., "Characteristics of solitary waves and weak double layers in the magnetospheric plasma," Phys. Rev. Lett., vol. 61, p. 82, 1988.

[5] R. Boström, "Observations of weak double layers on auroral field lines," in Abstracts Workshop Plasma Experiments in the Laboratory and in Space (Alpbach, Austria), July 1-5, 1991, p. 30.

[6] R. Boström, "Are weak double layers important for auroral plasma dynamics?" in Abstracts AGU Chapman Conf. Auroral Plasma Dynamics (convened by R. L. Lysak, Minneapolis), Oct. 21-25, 1991, p. 25; also discussion immediately following this talk, 1991.

[7] P. Coakley, N. Hershkowitz, R. Hubbard, and G. Joyce, "Experimental observations of strong double layers," Phys. Rev. Lett., vol. 40, p. 230, 1978.

[8] P. Coakley and N. Hershkowitz, "Laboratory double layers," Phys. Fluids, vol. 22, p. 1171, 1979.

[9] P. Coakley, L. Johnson, and N. Hershkowitz, "Strong laboratory double layers in the presence of a magnetic field," Phys. Lett., vol. 70A, p. 425, 1979.

[10] P. Coakley and N. Hershkowitz, "Moving double layers," Phys. Lett., vol. 83 A, p. 131,1981

[11] N. Hershkowitz, "Double layers and electrostatic shocks," J. Geophys. Res., vol. 86, p. $3307,1981$.

[12] N. Hershkowitz, G. L. Payne, and C. Chan, "Weak double layers," Plasma Phys., vol. 23, p. 903, 1981.

[13] C. Chan, N. Hershkowitz, and G. L. Payne, "Laboratory double layers with no external fields," Phys. Lett., vol. 83A, p. 328, 1981

[14] C. Chan, M. Khazei, K. E. Lonngren, and N. Hershkowitz, "Excitation of multiple ion acoustic shocks," Phys. Fluids, vol. 24, p. 1452, 1981.

[15] C. Chan, N. Hershkowitz, and K.E. Lonngren, "Electron temperature differences across double layers," in Proc. Symp. Plasma Double Layers (Riso, Denmark), Sept. 1982, Riso/Report R-472. 
[16] C. Chan and N. Hershkowitz, "Transition from single to multiple double layers," Phys. Fluids, vol. 25, p. 2135, 1982.

[17] C. Chan, N. Hershkowitz, and K. E. Lonngren, "Electron temperature differences and double layers," Phys. Fluids, vol. 26, p. 1587, 1983.

[18] C. Chan, M-H. Cho, N. Hershkowitz, and T. Intrator, "Laboratory evidence for ion-acoustic-type double layers," Phys. Rev. Lett., vol. 52, p. 1782,1984

[19] C. Chan, M-H. Cho, T. Intrator, and N. Hershkowitz, "Small amplitude double layers in laboratory plasmas," in Proc. Second Symp. Plasma Double Layers and Related Topics (Innsbruck, Austria), July 5-6, 1984, p. 200.

[20] C. Chan, M-H. Cho, N. Hershkowitz, and T. Intrator, "Experimental observation of slow ion acoustic double layers," in Phys. Rev. Lett., vol. 57 , p. $3050,1986$.

[21] A. Bailey III and N. Hershkowitz, "Three step double layers in the laboratory," Geophys. Res. Lett., vol. 15, pp. 99-102, 1988.

[22] T. Intrator, J. Menard, and N. Hershkowitz, "Multiple magnetized double layers in the laboratory," Phys. Fluids B, to be published.

[23] N. Hershkowitz, "Review of recent laboratory double layer experiments," Space Sci. Rev., vol. 41, pp. 351-391, 1985.

[24] J. E. Borovsky, "Parallel electric fields in extragalactic jets; double layers and anomalous resistivity in symbiotic reltaionships," Astrophys. $J$. vol. 306, p. 451,1986

[25] I. B. Bernstein, J. M. Greene, and M. D. Kruskal, Phys. Rev., vol. 108, p. $546,1957$.

26] G. Knorr and C. K. Goertz, Astrophys. Space Sci., vol. 31, p. 209, 1974

27] T. Intrator, C. Chan, N. Hershkowitz, and D. Diebold, "Nonlinear selfcontraction of electron waves," Phys, Rev. Lett., vol. 53, pp. 1233-1235, 1984.

[28] E.P. Szuszczewicz and J.C. Holmes, "Surface contamination of active electrodes in plasmas: distortion of conventional Langmuir probe measurements," J. Appl. Phys., vol. 46, pp. 5134-5139, 1975.

[29] J.C. Holmes and E.P. Szuszczewicz, "Plasma probe system with automatic sweep adjustment," Rev. Sci. Instrum., vol. 52, pp. 377-381, 1981.

[30] F. F. Chen, Plasma Diagnostic Techniques, R. H. Huddlestone and S. L. Leonard, Eds. New York: Academic Press, 1965, p. 134

[31] N. Hershkowitz and T. Christensen, "Plasma leaks to a blocked picket fence," Nucl. Fusion, vol. 17, p. 371, 1977.

[32] N. Hershkowitz, J. R. Smith, and H. Kozima, "Electrostatic self-plugging of a picket fence cusped magnetic field," Phys. Fluids, vol, 22, p. 122, 1979 .

[33] N. Hershkowitz, F. Ze, and H. Kozima, "Negative plasma potential produced by supplemental electron emission," Phys. Fluids, vol. 22 p. $338,1979$.

[34] N. Hershkowitz, J. R. Smith, and J. R. DeKock, "Spatial plasma potential variations in a multi-dipole device," Plasma Phys., vol. 21, p. 823, 1979.

[35] N. Hershkowitz, J. R. DeKock, P. Coakley, and S. L. Cartier, "Surface trapping of primary electrons by multi-dipole magnetic fields," Rev. Sci. Instrum., vol. 51, p. 64, 1980.

[36] N. Hershkowitz, J. R. DeKock, and C. Chan, "The effect of picket fence magnetic fields at the end of a magentized plasma," Nucl. Fusion, vol. 20 , p. $695,1980$.

[37] N. Hershkowitz and T. Intrator, "Improved source of cold plasma electrons and negative ions," Rev. Sci. Instrum., vol. 52, p. 1629, 1981.

[38] C. Chan, H. Bewley, N. Hershkowitz, and J. DeKock, "Picket fence suppression of secondary electrons," Nucl. Fusion, vol. 22, p. 105, 1982

[39] C. Chan, T. Intrator, and N. Hershkowitz, "The effect of secondary electrons on plasma potential in a multi-dipole device," Phys. Lett., vol. $91 \mathrm{~A}$, p. $167,1982$.

[40] N. Hershkowitz, K. Hendricks, and R. T. Carpenter, "Electrostatic plugging of leaks in a multi-dipole device," J. Appl. Phys., vol. 53, p. 4105,1982

[41] M-H. Cho, N. Hershkowitz, and T. Intrator, "Particle and balance of hotfilament discharge plasmas in a multidipole device," J. Apply. Phys. vol. 67, p. 7, 1990.

[42] T. Intrator, N. Hershkowitz, and C. Chan, "Experimental observations of nonlinearly enhanced $2 \omega_{\mathrm{C}} \mathrm{H}$ electromagnetic radiation excited by steady-state colliding electron beams," Phys. Fluids, vol. 27, p. 527, 1984

[43] B. Hultqvist, et al., "Simultaneous observation of upward moving field aligned energetic electrons and ions on auroral field lines," EOS Trans. Amer. Geophys. Union, vol. 69, p. 446, 1988.

[44] J.R. Smith, N. Hershkowitz, and P. Coakley, "The inflection poin method of interpreting emissive probe characteristics," Rev. Sci. In strum., vol. 50, p. 210, 1979.

[45] C. Chan, K.E. Lonngren, and N. Hershkowitz, "Deflected-beamexcited ion-cyclotron beam modes," IEEE Trans. Plasma. Sci., vol. 8 , pp. 512-517, 1980

[46] N. Hershkowitz, R. L. Goettsch, C. Chan, K. Hendricks, and R. T. Carpenter, "Detection of secondary electrons in a multi-dipole plasma," J. Appl. Phys., vol. 53, p. 5330, 1982.

[47] N. Hershkowitz, B. Nelson, J. Pew, and D. Gates, "Self-emissive probes," Rev. Sci. Instrum., vol. 54, p. 29, 1983

[48] E.Y. Wang, T. Intrator, and N. Hershkowitz, "A direct indication technique of plasma potential with differential emissive probe," Rev. Sci. Instrum., vol. 56, p. 519, 1985.

[49] E. Y. Wang, N. Hershkowitz, and T. Intrator, "Direct indication of plasma potential diagnostic based on secondary electron emission," Rev. Sci. Instrum., vol. 57, p. 1085, 1986.

[50] E. Y. Wang, N. Hershkowitz, T. Intrator, and C. Forest, "Technique for using emitting probes for potential measurements in rf plasmas," Rev. Sci. Instrum., vol. 57, p. 2425, 1986.

[51] E. Y. Wang et al., "Secondary electron emission-capactive probes for plasma potential measurements in the plasma with hot electrons," $J$ Appl. Phys., vol. 61, p. 4786, 1987.

[52] D. Diebold, N. Hershkowitz, A. D. Bailey III, M-H. Cho, and T. Intrator, "Emissive probe current bias method of measuring dc vacuum potential," Rev. Sci. Instrum., vol. 59, p. 270, 1988.

[53] C. H. Nam, N. Hershkowitz, M-H. Cho, T. Intrator, and D. Diebold "Multiple valued floating potentials of Langmuir probes," J. Appl. Phys. vol. 63, p. 5674, 1988.

[54] E.Y. Wang, et al. "An improved capacitive divider probe for plasma potential measurements in the Phaedrus-tokamak," Rev. Sci. Instrum., vol. 62, pp. 1494-1497, 1991.

[55] E. Y. Wang, W. Q. Li, S. W. Lam, and N. Hershkowitz, "Aperature effect on charged particles in a magnetized plasma," J. Appl. Phys., vol. 70, pp. 2570-2574, 1991.

[56] D. Diebold et al., "Space charge enhanced, plasma gradient induced error in satellite electric field measurements," J. Geophys. Res., to be published.

[57] M. H. Cho, C. Chan, N. Hershkowitz, and T. Intrator, "Measurement of vacuum space potential by an emissive probe," Rev. Sci. Instrum., vol. 55 , p. 631,1984

[58] N. Hershkowitz and M-H. Cho, "Measurement of plasma potential using collecting and emitting probes," J. Vacuum Sci. Tech.-A, vol. 6, p. 2054, 1988.

[59] N. Hershkowitz, M.H. Cho, C.H. Nam, and T. Intrator, "Langmuir probe characteristics in if glow discharges," Plasma Chemistry and Plasma Processing, vol. 8, pp. 35-52, 1988.

[60] N. Hershkowitz, Plasma Diagnostics, vol. 1, O. Aucielo and D. L. Flamm, Eds. San Diego, CA: Academic Press, 1989, ch. 3, pp. 113-184.

[61] D. Diebold, N. Hershkowitz, and T. Intrator, "Self-similar potential in the near wake," Phys. Fluids, vol. 30, p. 579, 1987.

[62] D. Diebold, N. Hershkowitz, and S. Eliezer, "Rarefaction shock in the near wake of an obstacle in a supersonic, two electron temperature plasma flow," Phys. Fluids, vol. 30, p. 3308, 1987.

[63] K. E. Lonngren and N. Hershkowitz, "A note on plasma expansion into a vacuum," IEEE Trans. Plasma Sci., vol. 7, p. 107, 1979.

[64] C. Chan et al, "Experimental observations of self-similar plasma expansion," Phys. Fluids, vol. 27, p. 266, 1984.

[65] T. Intrator et al., "The virtual cathode as a transient double sheath," $J$. Appl. Phys., vol. 64, pp. 2927-2933, 1988.

[66] P.G. Coakley and N. Hershkowitz, "Secondary electrons in a plasmawall sheath," Phys. Lett., vol. 78A, p. 145, 1980.

[67] S. Meassick, M-H. Cho, and N. Hershkowitz, "Measurement of plasma presheath," IEEE Trans. Plasma Sci., vol. 13, p. 115, 1985.

[68] M-H. Cho, N. Hershkowitz, and T. Intrator, "Temporal evolution of collisionless sheaths," J. Vacuum Sci. Tech. A, vol. 6, p. 2978, 1988.

[69] G-H. Kim, M-H. Cho, and N. Hershkowitz, "Measurement of sheath potential with DC magnetic field in if inductive discharge plasma," to be submitted.

[70] R. Chodura, Phys. Fluids; vol. 25, p. 1628, 1982

Photographs and biographies of the authors were not available at the time of publication. 